\title{
Definitions of Sexual Dysfunctions in Women and Men: A Consensus Statement From the Fourth International Consultation on Sexual Medicine 2015
}

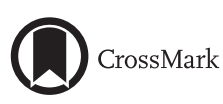

\author{
Marita P. McCabe, $\mathrm{PhD}^{1}$ Ira D. Sharlip, $\mathrm{MD}_{1}^{2}$ Elham Atalla, $\mathrm{MD}_{1}^{3}$ Richard Balon, $\mathrm{MD}_{r}^{4}$ \\ Alessandra D. Fisher, MD, PhD, ${ }^{5}$ Edward Laumann, $\mathrm{PhD}^{6}$ Sun Won Lee, MD, Ron Lewis, MD, ${ }^{8}$ and \\ Robert T. Segraves, $M D^{9}$
}

\begin{abstract}
Introduction: Definitions of sexual dysfunctions in women and men are critical in facilitating research and enabling clinicians to communicate accurately.

Aims: To present the new set of definitions of all forms of sexual dysfunction in women and men adopted by the Fourth International Consultation on Sexual Medicine (ICSM) held in 2015.

Methods: Classification systems, including the International Classification of Diseases, 10th Edition and the Diagnostic and Statistical Manual of Mental Disorders, Fifth Edition, and systems that focus on only specific types of sexual dysfunctions (eg, the International Society for Sexual Medicine definition for premature ejaculation) were reviewed.

Main Outcome Measures: Evidence-based definitions were retained, gaps in definitions were identified, and outdated definitions were updated or discarded. Where evidence was insufficient or absent, expert opinion was used. Some definitions were self-evident and termed clinical principles.
\end{abstract}

Results: The evidence to support the various classification systems was carefully evaluated. A more comprehensive analysis of this evidence can be found in two other articles in this journal that consider the incidence and prevalence and the risk factors for sexual dysfunction in men and women. These data were used to shape the definitions for sexual dysfunction that have been recommended by the 2015 ICSM.

Conclusion: The definitions that have been adopted are those that are most strongly supported by the literature at this time or are considered clinical principles or consensus of experts' opinions. As more research and clinical studies are conducted, there likely will be modifications of at least some definitions.

J Sex Med 2016;13:135-143. Copyright (c) 2016, International Society for Sexual Medicine. Published by Elsevier Inc. All rights reserved.

Key Words: International Consultation on Sexual Medicine; Definitions of Male and Female Sexual Dysfunction; Diagnostic and Statistical Manual of Mental Disorders, Fifth Edition; International Classification of Diseases, 10th Edition

Received September 30, 2015. Accepted December 20, 2015.

'Institute for Health and Ageing, Australian Catholic University, Melbourne, Australia;

${ }^{2}$ University of California, San Francisco, CA, USA;

${ }^{3}$ RCSI Medical University, Adilya, Bahrain;

${ }^{4}$ Department of Psychiatry and Behavioral Neurosciences, Wayne State University, Detroit, MI, USA;

${ }^{5}$ SODc Medicina della Sessualità e Andrologia, Florence, Italy;

${ }^{6}$ Department of Sociology, University of Chicago, Chicago, IL, USA;

${ }^{7}$ Department of Urology, Sungkyunkwan University School of Medicine, Seoul, Korea;

${ }^{8}$ Georgia Regents University, Augusta, GA, USA;

${ }^{9}$ Case Western Reserve University, Cleveland, $\mathrm{OH}$, USA

Copyright @ $\odot$ 2016, International Society for Sexual Medicine. Published by Elsevier Inc. All rights reserved.

http://dx.doi.org/10.1016/j.jsxm.2015.12.019

\section{INTRODUCTION}

This article describes the different classification systems for sexual dysfunction that have been used to guide research and practice in sexual medicine. Then, the definitions of sexual dysfunctions for men and women adopted by the Fourth International Consultation on Sexual Medicine (ICSM) are introduced. Draft definitions of these sexual dysfunctions and the rationale for their adoption were presented to the general meeting of the Fourth ICSM in June 2015. Comments from delegates were incorporated into a revised set of definitions, and then these revised definitions were presented to the chairs of the other ICSM committees. The final definitions in this article are those that were approved and adopted by the Fourth ICSM.

Commonly accepted diagnostic criteria influence how clinicians organize their thinking about clinical conditions, how 
clinical activity is coded for reimbursement, and how populations are defined in clinical research. Clinicians can use the experience of other clinicians only to the extent that they agree on the definition of the condition being studied or treated. Data from clinical trials and epidemiologic studies are relevant to the clinician only to the extent that they use similar definitions of the condition being treated by the clinician. Commonly accepted definitions permit global information exchange and communication among clinicians, their patients, and health care systems. These systems also facilitate the training of future clinicians and are used by other sectors such as health care policy makers, payers of health care, and pharmaceutical companies. ${ }^{1}$

Various approaches to taxonomy are used in medicine. A classic approach in medicine is to define a disease by its etiology. Such an approach applies in certain bacteriologic infections and nutritional deficiencies. ${ }^{2}$ However, the etiology of sexual dysfunctions is often unknown or presumed to be multifactorial. In such cases, a descriptive taxonomy is used. Most current classification systems for sexual dysfunctions are primarily descriptive. A problem with the most widely used current systems of classification is the assumption of mind vs body dualism, with disorders being classified as psychiatric or medical in etiology. ${ }^{3}$ This problem is perhaps best appreciated in the definition of lifelong female orgasmic disorder. Although current research indicates a clear genetic contribution to orgasmic function, psychosocial influences are perhaps of equal or greater importance. In most cases, one cannot easily separate organic from non-organic factors. Part of this separation of diagnostic systems can be understood in historical context. With some exceptions, most treatments of sexual disorders before the advent of the phosphodiesterase inhibitors were by psychiatrists or psychologists and it was commonly assumed that the vast majority of sexual disorders had psychological etiologies. ${ }^{4}$

\section{MAJOR CLASSIFICATION SYSTEMS}

Although different classification systems for sexual dysfunctions have been proposed, the International Classification of Diseases, 10th Edition (ICD-10 $)^{5}$ by the World Health Organization and the Diagnostic and Statistical Manual of Mental Disorders (fourth edition with text revision or fifth edition; $D S M-1 V-T R$ and $D S M-5)^{6,7}$ by the American Psychiatric Association have been the most widely used systems internationally. Although the DSM system is a psychiatric system, some of its diagnostic criteria have been adopted to define conditions that are assumed to have an organic etiology.

\section{INTERNATIONAL CLASSIFICATION OF DISEASES, 1OTH EDITION}

The ICD-10 was approved by the World Health Association in 1990 and published in $1992 .^{5}$ This system is in the process of revision. Completion of the ICD-11 is anticipated in 2017.
In the $I C D-10$, one codes disorders as organic or as non-organic. The organic sexual dysfunction codes are erectile dysfunction (ED), vaginismus, and dyspareunia of organic etiology. All organic codes are contained in the chapter on diseases of the genitourinary system. In contrast, there are 10 separate nonorganic sexual dysfunction codes, including lack of sexual desire, sexual aversion or lack of sexual enjoyment, failure of genital response, orgasmic dysfunction, premature ejaculation (PE), non-organic vaginismus, non-organic dyspareunia, excessive sexual drive, and two non-specific codes (similar to the "not otherwise specified" codes in the DSM-IV). The ICD-10 diagnostic codes for sexual dysfunctions not caused by organic disorders or disease and their descriptions are listed below.

\section{ICD-10 Sexual Dysfunction Not Caused by an Organic Disorder or Disease}

\section{Lack or Loss or Sexual Desire}

Loss of sexual desire is the principal problem and is not secondary to other sexual difficulties, such as erectile failure or dyspareunia. Lack of sexual desire does not preclude sexual enjoyment or arousal but makes the initiation of sexual activity less likely.

\section{Sexual Aversion}

The prospect of sexual interaction with a partner is associated with strong negative feelings and produces sufficient anxiety that sexual activity is avoided.

\section{Lack of Sexual Enjoyment}

Sexual responses occur normally and orgasm is experienced, but there is a lack of appropriate pleasure. This complaint is much more common in women than in men.

\section{Failure of Sexual Response}

In men, the principal problem is ED (ie, difficulty in developing or maintaining an erection suitable for satisfactory intercourse). If erection occurs normally in certain situations (eg, during masturbation or sleep or with a different partner), then the causation is likely to be psychogenic. Otherwise the correct diagnosis of non-organic ED could depend on special investigations (eg, of nocturnal penile tumescence) or the response to psychological treatment.

In women, the principal problem is vaginal dryness or failure of lubrication. The cause can be psychogenic or pathologic (eg, infection) or estrogen deficiency (eg, postmenopausal). It is unusual for women to complain primarily of vaginal dryness except as a symptom of postmenopausal estrogen deficiency.

\section{Orgasmic Dysfunction}

Orgasm does not occur or is markedly delayed. This might be situational (ie, occurs only in certain situations), in which case 
the etiology is likely to be psychogenic, or invariable, when physical or constitutional factors cannot be easily excluded except by a positive response to psychological treatment. Orgasmic dysfunction is more common in women than in men.

\section{Premature Ejaculation}

$\mathrm{PE}$ is the inability to control ejaculation sufficiently for both partners to enjoy sexual interaction. In severe cases, ejaculation might occur before vaginal entry or in the absence of an erection. $\mathrm{PE}$ is unlikely to be of organic origin but can occur as a psychological reaction to organic impairment (eg, erectile failure or pain). Ejaculation also can appear prematurely if erection requires prolonged stimulation, causing the interval from satisfactory erection to ejaculation to be shortened; the primary problem in such a case is delayed erection.

\section{Non-Organic Vaginismus}

Vaginismus is spasm of the muscles that surround the vagina, causing occlusion of the vaginal opening. Penile entry is impossible or painful. Vaginismus can be a secondary reaction to some local cause of pain, in which case this category should not be used.

\section{Dyspareunia}

Dyspareunia (pain during sexual intercourse) occurs in women and men. It often can be attributed to a local pathologic condition and then should be appropriately categorized. In some cases, no obvious cause is apparent and emotional factors might be important. This category is used only if there is no other more primary sexual dysfunction (eg, vaginismus or vaginal dryness).

\section{Excessive Sexual Drive}

Men and women occasionally complain of excessive sexual drive as a problem in its own right, usually during the late teenage period or early adulthood. When excessive sexual drive is secondary to an affective disorder or when it occurs during the early stages of dementia, then the underlying disorder should be coded.

\section{DIAGNOSTIC AND STATISTICAL MANUAL OF MENTAL DISORDERS, FIFTH EDITION}

The DSM published by the American Psychiatric Association has been translated into more than 20 languages and has had a major role in Europe and the United States in setting criteria for the diagnosis of mental disorders. Specific criteria for sexual dysfunctions were introduced in the DSM-III in 1980 and then modified in subsequent versions (DSM-III-R in 1987, DSM-IV in 1994, and DSM-IV-TR in 2000). The DSM-5 was introduced in 2013 .
The $D S M-5^{7}$ represents a major shift conceptually from the $D S M-I V-T R$ in several ways. The DSM-5, in contrast to earlier versions of the $D S M$, no longer requires a parallelism between the sexes and is no longer based on the sexual response cycle as proposed by Masters and Johnson. In contrast to the DSM-IV$T R{ }^{6}$ the $D S M-5$ specifies duration and severity (frequency) criteria for most disorders. Most sexual dysfunction diagnoses require that the problems have persisted for at least 6 months and occur on approximately $75 \%$ of sexual occasions to be diagnosed as sexual dysfunctions. The etiologic subtypes have been deleted in the DSM-5. Eight specific diagnoses are specified: delayed ejaculation, erectile disorder, female orgasmic disorder, female sexual interest-arousal disorder, genito-pelvic pain-penetration disorder, male hypoactive sexual desire disorder, premature (early) ejaculation, and substance- or medication-induced disorder. There also are two non-specific codes: other specified sexual dysfunction and unspecified sexual dysfunction. Sexual aversion disorder was deleted as a designated diagnosis because of its infrequent use and inconsistent definition. Genito-pelvic pain-penetration disorder combines elements of dyspareunia and vaginismus into a single diagnosis. The basis for this change is that most clinicians cannot reliably distinguish vaginismus from dyspareunia. Female hypoactive sexual desire disorder and female arousal disorder have been deleted from the DSM-5 and replaced by female sexual interest-arousal disorder, which combines elements of subjective sexual desire and arousal into one category. Female arousal disorder (defined in the DSM-IV$T R$ as the absence of genital arousal) was not included in the DSM-5, because absence of genital arousal is rarely identified independently of low sexual desire unless related to hypoestrogenism, which, by definition, is not a psychiatric diagnosis.

The DSM-5 definitions and criteria for sexual dysfunctions are included below. For each dysfunction, one should specify whether the dysfunction is:

- lifelong (the disturbance has been present since the individual became sexually active) or acquired (the disturbance began after a period of relatively normal sexual function)

- generalized (not limited to certain types of stimulation, situations, or partners) or situational (occurs only with certain types of stimulation, situations, or partners)

- mild, moderate, or severe in distress over symptoms in criterion A (a description of the distinguishing features of the dysfunction)

All disorders except genito-pelvic pain-penetration disorder require that the symptoms meet the DSM-5 definition of that condition (criterion A), have been present for 6 months on at least $75 \%$ of sexual occasions (criterion B), cause clinically significant distress (criterion $\mathrm{C}$ ), are not a consequence of a nonsexual mental disorder or of a severe relationship distress or other significant stressors, and are not attributable to the effect of a medication or illness (criterion D). 


\section{DSM-5 Definitions of Sexual Dysfunctions in Women}

\section{Female Sexual Interest-Arousal Disorder}

Lack of, or significantly decreased sexual interest or arousal is manifested by at least three of the following characteristics: (i) absent or decreased interest in sexual activity; (ii) absent or decreased sexual or erotic thoughts or fantasies; (iii) no or decreased initiation of sexual activity and typically unreceptive to a partner's attempts to initiate; (iv) absent or decreased sexual excitement or pleasure during sexual activity in almost all or all (approximately $75 \%$ $100 \%$ ) sexual encounters (in identified situational contexts or, if generalized, in all contexts); (v) absent or decreased sexual interest or arousal in response to any internal or external sexual or erotic cues (eg, written, verbal, or visual); or (vi) absent or decreased genital or non-genital sensations during sexual activity in almost all or all(approximately $75 \%-100 \%$ ) sexual encounters (in identified situational contexts or, if generalized, in all contexts).

\section{Female Orgasmic Disorder}

This disorder is manifested by either of the following symptoms and experiences on almost all or all (approximately 75\%$100 \%$ ) occasions of sexual activity (in identified situational contexts or, if generalized, in all contexts): (i) marked delay in, marked infrequency of, or absence of orgasm; or (ii) markedly decreased intensity of orgasmic sensations.

\section{Genito-Pelvic Pain-Penetration Disorder}

This disorder manifests as persistent or recurrent difficulties with at least one of the following: (i) vaginal penetration during intercourse; (ii) marked vulvovaginal or pelvic pain during vaginal intercourse or penetration attempts; (iii) marked fear or anxiety about vulvovaginal or pelvic pain in anticipation of, during, or as a result of vaginal penetration; or (iv) marked tensing or tightening of the pelvic floor muscles during attempted vaginal penetration.

\section{DSM-5 Definitions of Sexual Dysfunctions in Men}

\section{Male Hypoactive Sexual Desire Disorder}

This disorder is manifested by persistently or recurrently deficient (or absent) sexual or erotic thoughts or fantasies and desire for sexual activity. The judgment of deficiency is made by the clinician, taking into account factors that affect sexual functioning, such as age and general and sociocultural contexts of the individual's life.

\section{Erectile Disorder}

At least one of the following symptoms must be experienced on almost or all (approximately 75\%-100\%) occasions of sexual activity (in identified situational contexts or, if generalized, in all contexts): (i) marked difficulty in obtaining an erection during sexual activity, (ii) marked difficulty in maintaining an erection until completion of sexual activity, or (iii) marked decrease in erectile rigidity.

\section{Premature (Early) Ejaculation}

This disorder is defined as a persistent or recurrent pattern of ejaculation occurring during partnered sexual activity within approximately 1 minute after vaginal penetration and before the individual wishes it. Note: Although the diagnosis of premature (early) ejaculation can be applied to individuals engaged in nonvaginal sexual activities, specific duration criteria have not been established for these activities.

\section{Delayed Ejaculation}

Either of the following symptoms must be experienced on almost all or all occasions (approximately 75\%-100\%) of partnered sexual activity (in identified situational contexts or, if generalized, in all contexts) and without the individual desiring delay: (i) marked delay in ejaculation or (ii) marked infrequency or absence of ejaculation.

\section{DSM-5 Definitions of Sexual Dysfunctions That Can Occur in Men and Women}

\section{Substance- or Medication-Induced Sexual Dysfunction}

This dysfunction refers to a sexual disorder that is clearly etiologically related to a medication or substance.

\section{Other Diagnostic Systems}

A consensus group funded by the American Urological Association was formed to review existing criteria for female sexual dysfunctions. The American Foundation for Urologic Disease (currently known as the Urology Care Foundation) panel issued a formal recommendation in $2004 .^{8}$ One goal of this panel was to establish a set of criteria that would be used irrespective of etiology. In other words, conditions of organic and non-organic etiologies would be combined in the same diagnostic system. Desire disorders included lack of responsive desire. This was a major change in the description of sexual desire in women that was subsequently incorporated into the DSM-5. The model also emphasized that many sexually responsive women do not report spontaneous sexual desire and that a diagnosis of problematic lack of desire should not be made unless the clinician judged the lack of desire to be beyond normative lessening with lifecycle and relationship duration. Three separate types of female sexual arousal problems were distinguished: lack of subjective feelings of sexual arousal, lack of genital arousal, and lack of both types of arousal. Other diagnoses included dyspareunia, vaginismus, and orgasmic disorder. Persistent genital arousal also was included in their diagnostic categories. This refers to a condition in which persistent and unwanted genital arousal occurs in the absence of sexual stimulation. This particular diagnosis does not exist in the $D S M-5$ or $I C D-10$. This consensus document, particularly the definition of desire problems in women, had a major influence on thought in the field of female sexual dysfunction.

The International Society for Sexual Medicine (ISSM) has proposed an operational definition for lifelong and acquired PE. The 
ISSM definition of PE is a male sexual dysfunction characterized by ejaculation that always or nearly always occurs before or within approximately 1 minute of vaginal penetration from the first sexual experience (lifelong PE) or a clinically significant and bothersome decrease in latency time, often to approximately 3 minutes or less (acquired PE), the inability to delay ejaculation on all or nearly all vaginal penetrations, and negative personal consequences, such as distress, bother, frustration, and/or the avoidance of sexual intimacy. ${ }^{9}$ The ISSM definition of PE had an obvious influence on the delineation of the diagnosis of PE in the DSM-5.

Another major influence on the definitions of sexual dysfunction is the ICSM. The first consultation took place in 1999. The second consultation occurred in 2003 and the third in 2009. These consultations advocated a diagnostic system that could be applied independent of etiology. The female diagnoses resembled those described by the Urology Care Foundation panel and included a unisex definition of sexual interest-desire dysfunctions, genital arousal disorder, subjective arousal disorder, combined genital and subjective arousal disorder, persistent genital arousal disorder, dyspareunia, vaginismus, a unisex definition of sexual aversion, and women's orgasmic disorder. Disorders specific to men included ED, priapism, Peyronie disease, and orgasmic dysfunction, which included delayed ejaculation, $\mathrm{PE}$, orgasmic dysfunction, and anejaculation.

\section{DIAGNOSTIC CRITERIA IN EPIDEMIOLOGIC STUDIES}

There are inherent difficulties in gathering data in epidemiologic studies that can be meaningfully linked to clinically based definitions and classifications. Population-representative survey studies of sexual dysfunction must take into account time constraints on the interview instrument such that no more than three or four questions per dysfunction are feasible. In addition, if a study attempts to link sexual functionality to other features of the individual respondent, then it might not be possible to include as many as three to four questions per sexual difficulty. Thus, the use of a recent period for self-report of a particular sexual difficulty maximizes reliable recall of the frequency and severity of the difficulty within that period and, possibly, the degree of distress.

The period reported in epidemiologic studies needs to be reasonably short (ie, 1-3 months). A longer period requires the respondent to engage in even more complicated algorithms to estimate the frequency and severity of the difficulty. Complicated, unknowable, and non-comparable algorithms across respondents can render the data gathered meaningless. The problem of specifying the period is especially important in the context of age heterogeneity in the population surveyed. Younger persons will have higher rates of sexual frequency per unit of time than older persons. Thus, younger people typically will have a better basis for reliably estimating the frequency and severity of sexual difficulties than older persons. ${ }^{10}$ It is critical that anyone attempting to extrapolate data from epidemiologic studies to clinical contexts must be aware of the methodologic differences in how data are obtained in epidemiologic studies as opposed to clinical contexts.

In addition, it is worth cautioning about the pitfalls of attempting to infer population prevalence of specific sexual difficulties from samples gathered in clinical settings. The issue here is that there is simply no way of assessing the selection biases attendant on being included in a clinical sample that renders the clinical sample unrepresentative of the population at large. Put another way, clinical samples fundamentally differ from representative population samples in unknown ways and magnitudes.

There is a need to recognize that definitions for sexual dysfunction can differ for epidemiologic studies from those needed for clinical applications. Epidemiologic studies can accurately assess the incidence and prevalence of an identified problem in a community. However, the problem so identified might not correspond closely with the requirements to reach a clinical diagnosis. For example, a clinical diagnosis can have additional requirements, such as duration, severity, and clinically meaningful distress. It is difficult, if not impossible, for epidemiologic research to measure these additional variables accurately.

\section{DEFINITIONS OF SEXUAL DYSFUNCTIONS RECOMMENDED BY THIS CONSULTATION}

It is important to realize that currently there are two officially sanctioned systems with international influence, the DSM-5 and the $I C D-10$. The $I C D-10$ is focused on the definition of medical conditions and the DSM-5 is a document that primarily defines psychiatric conditions. As a result, there are inherent biases in the definitions from these two diagnostic systems. The DSM-5 was introduced in May 2013. Although it has been legitimately criticized, it is simply too soon after its introduction to judge its merits. The ICD-11 is in the process of modification as this consultation occurs. Thus, the diagnostic criteria of the ICD-11 are unknown. Multiple partially conflicting, partially overlapping definitions of sexual dysfunctions can be a real disservice to the field. For our knowledge base to increase, there needs to be some certainty that findings from different clinical and research sites are using similar populations of patients. The use of the same diagnostic label, but with differing definitions by different investigators, can impede progress, because one is never certain if the results reported by one center are applicable to other patient populations.

This leaves the question of whether the consultation should have endorsed an existing system or should have proposed yet another system. Despite the problems related to choosing a hybrid system of definitions taken from various sources, the consultation believes that for each sexual dysfunction, the most accurate and contemporary definition should be used. Therefore, the final recommendation of the consultation is to adopt 
some DSM-5 definitions, some DSM-1V-TR definitions, some $I C D-10$ definitions, and some new definitions developed by this consensus meeting. The consultation recognizes that some new definitions are not mature and might change in the future as new research and clinical experience accumulate and as there are improvements in study design. Many definitions do not have a sufficient evidence base to assign a reliable level of evidence or grade of recommendation. For definitions that have not been validated by objective data, the consultation believes that it is impossible to assign grades of recommendation, although it remains important to define these terms. In these cases for which grades of recommendations cannot be assigned, the terms expert opinion and clinical principle are used.

\section{Expert Opinion}

A statement achieved by panel consensus that is based on the panel members' clinical training, experience, knowledge, and judgment and for which there is no or insufficient published evidence.

\section{Clinical Principle}

A statement about clinical or research practice that is very widely agreed to by clinicians and/or researchers from several medical and/or scientific disciplines and for which there might or might not be evidence in the medical literature. (An example of a clinical principle is the concept that patients complaining of a medical problem should have a medical history and physical examination. Although there is no evidence to prove the concept, it is widely agreed that patients complaining of a medical problem should have a medical history and physical examination, making the concept a "clinical principle").

The method of assessing levels of evidence and strengths of recommendation was the method used throughout the Fourth ICSM. A level of evidence was assigned to each study reviewed. Strength of recommendation was based on all article making up the body of evidence on a specific topic.

Briefly, levels of evidence were based on the following system:

- Level 1 evidence was assigned to (i) meta-analyses of randomized controlled trials (RCTs), (ii) good-quality RCTs, or (iii) all or none studies in which no treatment is not an option.

- Level 2 evidence was assigned to (i) low-quality RCTs (eg, $<80 \%$ follow-up) or (ii) meta-analyses of good-quality prospective cohort studies.

- Level 3 evidence was assigned to (i) good-quality retrospective case-control studies in which a group of patients who have a condition are matched appropriately with control individuals who do not have the condition or (ii) good-quality case series in which a complete group of patients with the same condition, disease, or therapeutic intervention are described without a comparison control group.

- Level 4 evidence was assigned to studies based on expert opinion.
Briefly, strengths of recommendation were based on the following system:

- Grade A means that the statement is highly recommended. Grade A recommendation was assigned if there was consistent level 1 evidence. Grade A recommendation usually means that the statement is mandatory.

- Grade B means that the statement is recommended but not highly recommended. Grade B recommendation was assigned if there was consistent level 2 and/or level 3 evidence or if there was majority evidence from RCTs.

- Grade C means that the statement is optional. Grade C recommendation was assigned if there were level 4 studies or majority evidence from level 2 and/or level 3 studies or Delphi-processed expert opinion.

- Grade D means that the statement is not recommended or that no recommendation could be made. Grade D recommendation was assigned if there was inadequate or conflicting evidence or when expert opinion was delivered without a formal analytical process.

In the diagnosis of any sexual dysfunction, it should be noted whether the dysfunction:

1. Is lifelong or acquired

2. Has been in place for at least 3 months to be classified as a sexual dysfunction. For female genital and pelvic pain dysfunction, this period is 1 month. For ED due to radical pelvic surgery, this period is immediately after surgery. These periods are based on research findings and clinical opinion. Although it could be argued that the periods could be different and are somewhat arbitrary, these periods allow for early intervention before the dysfunction become entrenched.

3. Leads to individual distress. Without an experience of distress, sexual dysfunction should not be diagnosed.

4. Occurs in $75 \%$ to $100 \%$ of sexual experiences

\section{DEFINITIONS OF SEXUAL DYSFUNCTIONS THAT OCCUR IN WOMEN}

In relation to the definitions of sexual dysfunctions that occur in women, the definitions have been drawn from several classification systems. There was consensus by the ICSM on the definitions described in this article. Thus, the input into these final definitions has been drawn from a large number of international experts in the field of sexual dysfunction.

Members of the Fourth ICSM were of the view that hypoactive sexual desire dysfunction should be kept as a separate entity from female sexual arousal dysfunction. Data on the incidence and prevalence and risk factors for sexual dysfunction indicate clearly that the separation of these two dysfunctions is supported by the current epidemiologic and risk factor literature. Further research is necessary to determine the extent to which these two dysfunctions are separate entities. This decision is at variance with the DSM-5, which combined these two 
dysfunctions into a single disorder. We expect that over the course of the next few years, research will clarify the best way of conceptualizing these two female sexual dysfunctions.

The literature on the incidence and prevalence and risk factors for female sexual dysfunction indicates a high level of overlap in the experience of problems with desire, arousal, and orgasm for women. However, the disorders are experienced differently, with desire problems appearing to be the most prevalent disorder, followed by arousal and orgasm problems. ${ }^{11}$ For risk factors, many biological risk factors seem to be related to disorders of desire and arousal, but the role of biological risk factors for orgasm disorder is less clear. However, the psychological and sociocultural risk factors appear to be more likely related to levels of sexual interest. ${ }^{12}$

Substantially more research on the prevalence of female sexual dysfunction and the factors related to this dysfunction will greatly assist in determining the best way to conceptualize these dysfunctions. This is particularly the case for the emerging aspects of sexual dysfunction that are currently not well represented in classification systems. For example, further research is needed on persistent genital arousal disorder. Although the ICD10 includes excessive sexual drive as a disorder for men and women, there has been limited research on the nature of this complaint. ${ }^{13}$

\section{Hypoactive Sexual Desire Dysfunction}

Persistent or recurrent deficiency or absence of sexual or erotic thoughts or fantasies and desire for sexual activity (clinical principle).

\section{Female Sexual Arousal Dysfunction}

Persistent or recurrent inability to attain or maintain arousal until completion of the sexual activity, an adequate subjective assessment of her genital response (clinical principle).

\section{Female Orgasmic Dysfunction}

(i) Marked delay in, marked frequency of, or absence of orgasm and/or (ii) markedly decreased intensity of orgasmic sensation (grade B).

\section{Female Genital-Pelvic Pain Dysfunction}

Persistent or recurrent difficulties with at least one of the following: (i) vaginal penetration during intercourse; (ii) marked vulvovaginal or pelvic pain during genital contact; (iii) marked fear or anxiety about vulvovaginal or pelvic pain in anticipation of, during, or as a result of genital contact; or (iv) marked hypertonicity or overactivity of pelvic floor muscles with or without genital contact.

Some research and clinical studies have separated vaginismus from sexual pain disorder. Further research is needed to clarify this definition (grade $\mathrm{C}$ ).

\section{Persistent Genital Arousal Disorder}

Spontaneous, intrusive, and unwanted genital arousal (ie, tingling, throbbing, pulsating) in the absence of sexual interest and desire. Any awareness of subjective arousal is typically, but not invariably, unpleasant. The arousal is unrelieved by at least one orgasm and the feeling of arousal persists for hours or days (expert opinion).

\section{Postcoital Syndrome (Post-Orgasmic Illness Syndrome]}

Negative feelings, experiences, and/or physical symptoms such as headache, malaise, fatigue, and other symptoms after sexual activity (expert opinion).

\section{Hypohedonic Orgasm}

Lifelong or acquired decreased or low level of sexual pleasure with orgasm (expert opinion).

\section{Painful Orgasm}

The occurrence of genital and/or pelvic pain during or shortly after orgasm (expert opinion).

\section{DEFINITIONS OF SEXUAL DYSFUNCTIONS THAT OCCUR IN MEN}

Each definition was chosen for being the closest reflection of current evidence or expert opinion. Some definitions in this list are identical to definitions used in previous ICSMs, the ICD-10, the DSM-5, or other existing sources. Some definitions are modifications of previous definitions. The list also contains several new terms that have not been formally defined previously. Each definition was chosen for being the closest reflection of current evidence or expert opinion. The Fourth ICSM recommended that definitions of all known types of sexual dysfunction in men should be established to standardize research and practice and facilitate conversation on these conditions. The presence at the consultation of most of the world's experts on these conditions provided a unique opportunity to establish definitions for the full range of sexual dysfunctions in men.

\section{Male Hypoactive Sexual Desire Disorder}

Persistent or recurrent deficiency or absence of sexual or erotic thoughts or fantasies and desire for sexual activity (clinical principle).

\section{Erectile Dysfunction}

Consistent or recurrent inability to attain and/or maintain penile erection sufficient for sexual satisfaction (clinical principle).

\section{Premature Ejaculation}

A male sexual dysfunction characterized by ejaculation that always or nearly always occurs before or within approximately 1 
minute of vaginal penetration from the first sexual experience (lifelong PE) or a clinically significant and bothersome decrease in latency time, often to approximately 3 minutes or less (acquired $\mathrm{PE}$ ), the inability to delay ejaculation on all or nearly all vaginal penetrations, and negative personal consequences, such as distress, bother, frustration, and/or the avoidance of sexual intimacy (grade A).

Premature ejaculation is the preferred term and should be used to describe this condition rather than early ejaculation, rapid ejaculation, early orgasm, rapid orgasm, and other terms (expert opinion).

\section{Primary Delayed Ejaculation}

A lifelong experience of inability to ejaculate in all or almost all (75\%-100\%) occasions of coital activity, which causes distress.

Voluntary cessation of coital activity subsequently occurs after a variable time to avoid frustration, physical exhaustion, or genital irritation of self and/or partner.

Ejaculation might or might not occur by subsequent noncoital activity, including masturbation (grade E).

\section{Acquired Delayed Ejaculation}

A distressing lengthening of ejaculatory latency that occurs in most $(>50 \%)$ coital experiences after a period of normal ejaculatory function and/or a clinically meaningful change that results in distress. Voluntary cessation of coital activity subsequently occurs after a variable period to avoid further distress.

Ejaculation might or might not occur by subsequent noncoital activity, including masturbation.

Anejaculation occurs independent of the sensation of an orgasm (clinical principle).

\section{Retrograde Ejaculation}

Expulsion of seminal fluid into the bladder because of bladder neck dysfunction in the presence of otherwise normal emission and expulsion. There can be no or small amounts of antegrade ejaculation. Retrograde ejaculation is defined independently from the sensation of orgasm (clinical principle).

\section{Anejaculation}

The absence of normal antegrade ejaculation during orgasm owing to the absence of the emission and expulsion phases of the ejaculation reflex (clinical principle).

\section{Anhedonic Ejaculation}

Ejaculation without the pleasurable sensation of orgasm (clinical principle).

\section{Anorgasmia}

The inability to reach orgasm despite adequate and prolonged sexual stimulation leading to adequate sexual arousal. Anorgasmia might or might not lead to personal distress (grade C).

\section{Hypohedonic Orgasm}

Lifelong or acquired decreased or low level of sexual pleasure with orgasm (clinical principle).

\section{Painful Ejaculation or Orgasm}

The occurrence of genital and/or pelvic pain during or shortly after ejaculation or orgasm (clinical principle).

\section{Post-Orgasmic Illness Syndrome}

Flulike incapacitating physical and mental symptoms occurring within a few minutes to a few hours after an ejaculation, which usually last 3 to 7 days (expert opinion).

\section{Provisional Diagnoses of Restless Genital Syndromes}

Restless genital syndrome in men is characterized by persistent, disturbing penile sensations of ejaculatory urgency in the absence of erection, sexual desire, and/or sexual arousal. Restless genital syndrome can be accompanied by restless legs and/or complaints of overactive bladder and often leads to distress (grade C).

Restless genital syndrome in women is characterized by persistent, disturbing genital sensations often of orgasmic urgency in the absence of sexual desire and/or sexual arousal. Restless genital syndrome can be accompanied by restless legs and/or complaints of an overactive bladder and often leads to distress (grade C).

These definitions are offered as provisional and tentative. There is controversy about whether these rare syndromes are separate and identifiable from other sexual or psychiatric dysfunctions. Research is needed to clarify the nature of these syndromes.

\section{CONCLUSION}

These definitions were adopted by the Fourth ICSM in 2015. This consultation was comprised of many of the top international experts in the field of male and female sexual dysfunction. The definitions have many levels of empirical support, and some are based on expert clinical opinion, rather than a strong research base. However, these definitions were seen to represent the most up-to-date reflections of the definitions for sexual dysfunction. It is imperative that research be conducted to determine the support for these definitions and that, where necessary, appropriate modifications are made to reflect these research findings. 
Corresponding Author: Marita P. McCabe, PhD, Institute for Health and Ageing, Australian Catholic University, Level 6, 215 Spring Street, Melbourne, Victoria 3000, Australia. Tel: +61 39953 3602; E-mail: Marita.McCabe@acu.edu.au

Conflict of Interest: The authors report no conflicts of interest.

Funding: None.

\section{REFERENCES}

1. Segraves R, Balon R, Clayton A. Proposal for changes in diagnostic criteria for sexual dysfunctions. J Sex Med 2007; 4:567.

2. Zachar P, Kendler KS. Psychiatric disorders: a conceptual taxonomy. Am J Psychiatry 2007; 164:557.

3. Hatzimouratidis K, Hatzichristou D. Sexual dysfunctions: classifications and definitions. J Sex Med 2007; 4:41.

4. Bancroft J. The endocrinology of sexual arousal. J Endocrinol 2005; 186:411.

5. World Health Organization. International statistical classification of diseases and related health problems. 10th ed. Geneva: World Health Organization; 1992.
6. American Psychiatric Association. Diagnostic and statistical manual of mental disorders. 4th ed, text revision. Washington, DC: American Psychiatric Press; 2000.

7. American Psychiatric Association. Diagnostic and statistical manual of mental disorders. 5th ed. Washington, DC: American Psychiatric Press; 2013.

8. Basson R, Leiblum S, Brotto $L$, et al. Revised definitions of women's sexual dysfunction. J Sex Med 2004; 1:40.

9. Serefoglu EC, McMahon CG, Waldinger MD, et al. An evidencebased unified definition of lifelong and acquired premature ejaculation: report of the Second International Society for Sexual Medicine Ad Hoc Committee for the Definition of Premature Ejaculation. J Sex Med 2014; 17:1423.

10. Laumann E, Gagnon J, Michael R, et al. The social organization of sexuality. Sexual practices in the United States. Chicago: University of Chicago Press; 1994.

11. McCabe MP, Sharlip ID, Lewis R, et al. Incidence and prevalence of sexual dysfunction in men and women. J Sex Med 2015.

12. Sharlip ID, McCabe MP, Lewis R, et al. Risk factors for sexual dysfunction among women and men. J Sex Med 2015.

13. Facelle TM, Sadeghi-Nejad H, Goldmeier D. Persistent genital arousal disorder: characterization, etiology, and management. J Sex Med 2013; 10:439. 